\title{
The grey extinction of the ionizing cluster in NGC 3603 from ultraviolet to optical wavelengths
}

\author{
Xiaoying Pang ${ }^{1,2,3,4}$, Anna Pasquali ${ }^{3}$, Eva K. Grebel ${ }^{3}$ \\ ${ }^{1}$ Shanghai Institute of Technology, 100 Haiquan Road, Fengxian district, Shanghai 201418, China \\ ${ }^{2}$ National Astronomical Observatories, Chinese Academy of Sciences, 20A Datun Road, Chaoyang District, \\ 100012 Beijing, China \\ ${ }^{3}$ Astronomisches Rechen-Institut, Zentrum für Astronomie der Universität Heidelberg, Mönchhofstr. 12-14, \\ 69120 Heidelberg, Germany \\ ${ }^{4}$ Key Lab for Astrophysics, 100 Guilin Road, Shanghai 200234, China \\ xypang@bao.ac.cn
}

\begin{abstract}
We use photometry in the F220W, F250W, F330W, F435W filters from the High Resolution Channel of the Advanced Camera for Surveys and photometry in the $5555 \mathrm{~W}, \mathrm{~F} 675 \mathrm{~W}$, and $\mathrm{F} 814 \mathrm{~W}$ filters from the Wide Field and Planetary Camera 2 aboard the Hubble Space Telescope to derive individual stellar reddenings and extinctions for stars in the HD 97950 cluster in the giant H II region NGC 3603. The mean line-of-sight reddening for about a hundred main-sequence member stars inside the cluster is $E(F 435 W-F 555 W)=1.33 \pm 0.12$ mag. After correcting for foreground reddening, the total to selective extinction ratio is $R_{F 555 W}=3.75 \pm 0.87$ in the cluster. Within the standard deviation associated with $E(\lambda-\mathrm{F} 555 \mathrm{~W}) / \mathrm{E}(\mathrm{F} 435 \mathrm{~W}-\mathrm{F} 555 \mathrm{~W})$ in each filter, the cluster extinction curve at ultraviolet wavelengths tends to be greyer than the average Galactic extinction laws from Cardelli et al. (1989) and Fitzpatrick et al. (1999). It is closer to the extinction law derived by Calzetti et al. (2000) for starburst galaxies, where the $0.2175 \mu \mathrm{m}$ bump is absent. This indicates an anomalous extinction in the HD 97950 cluster, which may due to the clumpy dust distribution within the cluster, and the size of dust grains being larger than the average Galactic ISM.
\end{abstract}

Subject headings: HII regions - open clusters and associations: individual (NGC 3603) - ISM: dust, extinction - stars: massive - stars: winds, outflows

\section{Introduction}

The dependence of dust extinction on wavelength is described by the so-called extinction curve. In most regions of the Milky Way, the extinction curve rises like a power law from infrared (IR) to optical wavelengths, then shows a prominent bump at $0.2175 \mu \mathrm{m}$, and may steeply rise in the far ultraviolet (UV) (Figure 1). Possible contributors to the UV bump are interstellar graphite grains (Mathis et al. 1977), polycyclic aromatic hydrocarbon (PAH) molecules (Draine 1989) molecules, or $\mathrm{MgO}$ particles with a thin $\mathrm{O}^{2-}$ coating (Maclean et al. 1982).
A typical way to determine the interstellar extinction is the standard "pair method", which matches the spectral features of reddened mainsequence (MS) stars with identical unreddened MS standard stars (Massa et al. 1983). The extinction is derived for each star in terms of $A_{\lambda} / A_{V}$ or $E(\lambda-V) / E(B-V)$ as a function of inverse wavelength (Cardelli et al. 1988, 1989; Fitzpatrick 1999). Recently Fitzpatrick \& Massa (2005) introduced a new technique that derives the interstellar extinction curve from the UV to the IR by modeling the observed spectral energy distribution of reddened early-type stars.

The Galactic extinction curve has been exten- 
sively studied in a large number of papers (e.g., Seaton 1979; Savage et al. 1985; Cardelli et al. 1989, 1988; Fitzpatrick 1999; Valencic et al. 2004; Fitzpatrick \& Massa 2007, 2009), which have shown it to vary considerably among different lines of sight. The individual extinction curves obtained for various lines of sight are averaged to obtain the mean Galactic extinction curve, which has an average total to selective extinction ratio of $R_{V}=3.1$, and whose dependence on wavelength is parameterized with analytical functions. While the extinction curve at optical wavelengths does not seem to change much among different lines of sight, large variations exist in the UV range (Massa et al. 1983; Fitzpatrick 1999). The large rms dispersion associated with the different average Galactic extinction curves makes them almost indistinguishable from each other (Seaton 1979; Savage et al. 1985; Cardelli et al. 1989; Fitzpatrick 1999; Valencic et al. 2004; Fitzpatrick \& Massa 2007). We should bear in mind that any mean extinction curve is biased by the sample of lines of sight from which it was derived, i.e., different lines of sight probe different conditions of the interstellar medium (ISM).

However, the mean Galactic extinction curve does not work for other galaxies, especially not for starburst galaxies, where the star formation rate is a few tens of $\mathrm{M}_{\odot} \mathrm{yr}^{-1}$, and the total to selective extinction ratio $R_{V}$ is much larger $(\sim 4.05$; Calzetti et al. 1994, 1997, 2000) than in the diffuse Galactic ISM $\left(R_{V}=3.1\right)$. The extinction curve of starburst galaxies is grey and lacks a bump at $0.2175 \mu \mathrm{m}$ (Figure 1). The value of $R_{V}$ is usually treated as an indicator for the grain size (Cardelli et al. 1989). The higher than normal value of $R_{V}$ and the absence of a UV bump may imply different processing histories of dust grains in the ISM of starburst galaxies, which favor formation of large grains.

According to Calzetti et al. (1994), young massive stars are associated with dustier regions than the older and less massive stars. The large optical depth associated with young massive stars preserves the large dust grains, which absorb less UV light than smaller grains and thus may explain the absence of the UV bump in the extinction curve of starburst regions/galaxies (Pierini et al. 2005; Panuzzo et al. 2007). Furthermore, the strong UV radiation and stellar winds of massive stars are likely to destroy dust grains and change the properties of the local ISM. The stellar feedback from young massive stars produces an inhomogeneous, clumpy distribution of dust within star-forming regions, which cannot be represented with the uniform dust screen model commonly used for the Milky Way. A clumpy distribution of dust is effective in redirecting the scattered photons along the line of sight (Calzetti et al. 1994). In contrast, in the diffuse Galactic ISM the distant dust forms a uniform foreground extinction screen, through which absorption and the scattering remove flux from the line of sight. Therefore the extinction curve of starburst galaxies is greyer than the average Galactic extinction curve.

Unfortunately, most of the starburst galaxies are too distant to study their ISM with high angular resolution data. However, in the Milky Way, the environment of an $\mathrm{H}$ II region hosting a massive young cluster may resemble that of starburst galaxies. A systematic investigation of the reddened stars within an $\mathrm{H}$ II region can probe the effect of the presence of massive stars on dust grains, and extend our knowledge of the ISM in starburst regions from parsec to kiloparsec scales.

There is an ideally suited Galactic HII region hosting a starburst cluster of which highresolution and multi-band data are available. This is NGC 3603, a giant H II region containing the central ionizing cluster HD 97950 with a mass of $\sim 10^{4} \mathrm{M}_{\odot}$ (Harayama et al. 2008). A larger than normal total to selective extinction $R_{V}=4.3$ was found among stars in NGC 3603 that are brighter than $V=17 \mathrm{mag}$ and located at distances larger than $0.5 \mathrm{pc}$ from the HD 97950 cluster (Pandey et al. 2000). Sung \& Bessell (2004) derived a smaller $R_{V}$ value, $3.55 \pm 0.12$, from stars with $V \leq 16.5 \mathrm{mag}$ that are located at projected cluster-centric radii of $r \geq 0.4 \mathrm{pc}$. Values of $R_{V}$ larger than 3.1 were also found in a number of other young Galactic star clusters in $\mathrm{HII}$ regions, e.g., NGC 6530 (Fernandes et al. 2012); M 16, M 17, and NGC 6357 (Chini \& Krügel 1983; Chini \& Wargau 1990). A larger value of $R_{V}$ can be due to significant changes in dust properties, such as evaporation of small grains by the radiation of hot stars (Draine 2009) and growth of large grains (Cardelli et al. 1989; Hirashita 2012).

The strong radiation field of the OB stars in the HD 97950 cluster (Drissen et al. 1995; Melena 
et al. 2008) ionizes and sweeps away the ISM, and generates a cavity around the cluster where the gas reddening is the lowest (Pang et al. 2011). A shell structure was found at $1.2 \mathrm{pc}$ west of the cluster (Figure 2, Clayton 1986; Pang et al. 2011), which expands with a velocity of $55 \mathrm{~km} \mathrm{~s}^{-1}$. The engine of the expanding shell may be the stellar winds and the radiation pressure of the massive stars in the cluster. Such energy input into the ISM can change the dust grain properties significantly. Weingartner \& Draine (2001) found that when grains larger than $0.1 \mu \mathrm{m}$ are exposed to the radiation of $\mathrm{OB}$ stars, they are subjected to the photodesorption force, applied by a radiation field that is anisotropic because of a spatially uneven distribution of stars. Under this force, the large grains decouple from the gas and move away. Furthermore, the large grains may be destroyed or reduced to $75 \%$ of their initial radius by a shock speed as low as $40 \mathrm{~km} \mathrm{~s}^{-1}$, which can be generated by supernovae (Seab \& Shull 1983) or by the radiation pressure and the stellar winds of OB stars in a cluster. The large grains are destroyed or sputter into small grains and are pushed away. This agrees with the observation of Lebouteiller et al. (2007) that the emission of very small grains whose size is smaller than $0.02 \mu \mathrm{m}$ (Wood et al. 2008) increases from the cluster center outwards and peaks at cluster-centric distances of $1-1.5 \mathrm{pc}$.

Spectroscopy is only available for 26 early-type stars in NGC 3603 (Moffat 1983; Drissen et al. 1995; Melena et al. 2008). Using $U B V$ photometry, Pandey et al. (2000) derived stellar reddenings and extinctions for 51 probable member stars, whose memberships were inferred via photometric methods and which are mainly located in the outskirts of the cluster HD 97950 in NGC 3603. Sung \& Bessell (2004) extended the number of stars with stellar reddening $E(B-V)$ in NGC 3603 to a few hundreds. No membership probability estimation is available for these stars.

In our work, we obtain reddenings and extinctions for about a hundred MS member stars in HD 97950. The membership of these stars was determined via relative proper motions in Pang et al.(2013). We also derive the average extinction curve from UV to optical wavelengths for the member stars within the HD 97950 cluster, and compare it to the extinction laws in the Milky Way and in starburst galaxies.
In Section 2 we will introduce the observations and data reduction. We will discuss the individual stellar reddening of cluster stars in Section 3. We discuss the total to selective extinction ratio within the HD 97950 cluster in Section 4. A discussion of the inferred dust growth history and the extinction curve is presented in Section 5. Finally, we summarize our results in Section 6.

\section{Observations and Data reduction}

The HD 97950 cluster and its immediate surroundings in NGC 3603 were observed with the Hubble Space Telescope (HST). The UV data were taken with the High Resolution Channel (HRC) of the Advanced Camera for Surveys (ACS) in 2005 (GO 10602, PI: Jesús Maíz Apellániz) through the F220W, F250W, F330W, and F435W filters. The $\mathrm{HRC}$ is characterized by a spatial resolution of $0.03^{\prime \prime}$ pixel $^{-1}$ and a field of view of $29^{\prime \prime} \times 25^{\prime \prime}$. The optical observations were carried out with the Wide Field and Planetary Camera 2 (WFPC2) in two epochs: 1997 (GO 6763, PI: Laurent Drissen) and 2007 (GO 11193, PI: Wolfgang Brandner) through the $F 555 \mathrm{~W}, \mathrm{~F} 675 \mathrm{~W}$, and $F 814 \mathrm{~W}$ filters. The Planetary Camera (PC) chip was centered on the cluster $\left(0.045^{\prime \prime}\right.$ pixel $\left.^{-1}, 40^{\prime \prime} \times 40^{\prime \prime}\right)$ for both programs. The wavelength range of these filters is shown in Figure 1. The F220 W filter covers the UV bump in the extinction curve, which can effectively distinguish a normal from a grey extinction curve. Pang et al. (2013) reduced the two-epoch WFPC2 data and identified more than 400 member stars on the PC chip via relative proper motions. Of these member stars, 142 are in common between the HRC and PC images and thus have UV and optical photometry available (see Table $1)$.

The ACS/HRC data were reduced using Dolphot (Dolphin 2005), a stellar photometry package that was adapted from HSTphot (Dolphin 2000) to handle ACS images. We used the ACS module in Dolphot for the F220W, F250W, F330W and $F 435 \mathrm{~W}$ data. All the data consist of four dithered images. The exposure time for each single exposure image (*.flt) is $357 \mathrm{~s}$ in the F220 W, $44 \mathrm{~s}$ in the $F 250 \mathrm{~W}, 10 \mathrm{~s}$ in the $F 330 \mathrm{~W}$ and $2 \mathrm{~s}$ in the $\mathrm{F} 435 \mathrm{~W}$ filter. We masked bad and saturated pixels in the "flt" images with the routine ACSMASK. The offsets between dithered images were calculated with 
a python script. The photometry was produced by the routine Dolphot, with the specific parameters suggested for ACS data (Dolphin 2006). Dolphot performs point spread function (PSF) photometry with the precomputed PSF of each filter.

The WFPC2 data were reduced with HSTphot (Dolphin 2000), which uses a similar reduction procedure as Dolphot. A detailed description of the data reduction of the optical WFPC2 data is provided in Pang et al. (2013).

\section{Individual stellar reddenings in the HD 97950 cluster}

It is crucial to know which stars are cluster members in order to estimate the reddening of a given star cluster (e.g., Yadav \& Sagar 2001). Among the HD 97950 cluster member stars determined from relative proper motions (Pang et al. 2013, Table 2), there are five MS stars located in the cluster with projected distances of $r<0.7 \mathrm{pc}$ from the center, for which there are also spectral types available from Table 3 of Melena et al. (2008). We indicate the locations of these five stars (red circles) in the cluster in Figure 2. The photometry of these five MS stars is presented in Table 2.

In order to obtain the reddening of these five stars, we derive their intrinsic colors from an empirical zero age main sequence (ZAMS) according to their spectral types. The MS stars within the HD 97950 cluster were found to be consistent with a single burst population with an age of $1 \mathrm{Myr}$ (e.g., Kudryavtseva et al. 2012; Pang et al. 2013). Hence a ZAMS provides a good approximation of their properties in color-magnitude space. We use the empirical ZAMS from Sparke \& Gallagher (S\&G, 2007, their Table 1.4), which is based on a large number of nearby MS stars with reliably determined photometry, distances, and well-defined spectral types. The S\&G ZAMS only provides $U B V$ photometry in the Johnson filter system.

On the other hand, the Marigo et al. (2008) theoretical isochrones provide magnitudes not only in the standard Johnson filter system, but also in the ACS/HRC and WFPC2 systems, which are appropriate for the present data sets. These isochrones extend to $90 \mathrm{M}_{\odot}$, which is massive enough to cover the most massive member stars used in this paper. Hence we derive the corresponding F220W,
F250W, F330W, F435W, F555W, F675W, and $F 814 W$ magnitudes for each spectral type from a Marigo et al. (2008) 1 Myr-old isochrone of solar metallicity. This is easily doable since the Marigo et al. isochrones of a given age and metallicity use exactly the same luminosity, temperature, and mass steps for each photometric system. We can now plot two-color diagrams corresponding to the traditional $U B V$ two-color diagram, and obtain the appropriate reddening values for our five stars (Figure 3) by subtracting the intrinsic colors (blue points) from the observed colors (red points).

Repeating this procedure for different color combinations allows us to calculate the color excesses $E(F 220 W-F 434 W), \quad E(F 250 W-$ $F 435 W), \quad E(F 330 W-F 435 W), \quad E(F 435 W-$ $F 555 W), E(F 555 W-F 675 W)$, and $E(F 555 W-$ $F 814 W)$, and the extinctions $A_{F 220 W}, A_{F 250 W}$, $A_{F 330 W}, A_{F 435 W}, A_{F 555 W}, A_{F 675 W}$, and $A_{F 814 W}$ by comparing the intrinsic colors and magnitudes obtained from the ZAMS using the observed apparent magnitudes and a distance modulus of $14.2 \mathrm{mag}$ from Sung \& Bessell (2004).

The $(F 555 W-F 814 W)$ vs. F555W color-magnitude diagram (Figure 4) shows the member stars of the HD 97950 cluster based on the membership determinations by Pang et al. (2013). Black dots denote stars detected both in the optical and in the UV data. Open circles show those stars with optical detections only. Member stars with UV and optical photometry delineate the massive MS of the HD 97950 cluster. The fainter stars with optical detections only are either MS stars or premain-sequence (PMS) stars.

Note the wide "turn-on" transition region where PMS stars are moving towards the MS in the color range $(F 555 W-F 814 W)=1.8-2.6 \mathrm{mag}$. MS stars located in this overall region are contaminated with PMS stars. Some of the brightest PMS stars are also detected in our UV data (and accordingly are marked with black dots). Since our procedure to compute individual reddening values relies on MS stars, we exclude PMS stars from our analysis. Moreover, calculating reddenings for PMS stars is fraught with uncertainties due to the unknown effects caused by their circumstellar disk or dust shell absorbing the UV radiation, the inclination of such disks, accretion, etc (Baraffe et al. 2009).

In order to obtain a clean sample of MS 
stars, we consider only stars bluer than $(F 555 \mathrm{~W}$ $F 814 W)=1.8 \mathrm{mag}$ to be MS stars. The lowest stellar mass of these objects corresponds to $\sim 4 \mathrm{M}_{\odot}$. Altogether, we select 111 MS member stars to study the reddening and extinction curve within the cluster.

We use the mean reddening vector $E(F 330 W-$ $F 435 W) / E(F 435 W-F 555 W)=0.984 \pm 0.014$ (the error is the standard deviation) of the five MS stars selected from Melena et al. (2008) to project the 111 member MS stars onto the $1 \mathrm{Myr}$-old Marigo et al. (2008) isochrone in color-color space and derive their individual $E(F 330 W-F 435 W)$ and $E(F 435 W-F 555 W)$ (Figure 5$)$. The uncertainty of the color excess comes from the uncertainty in the reddening vector and the photometry. The reddening vector contributes $\sim 0.05 \mathrm{mag}$ uncertainty to the value of $E(F 330 W-F 435 W)$, $\sim 0.01 \mathrm{mag}$ to $E(F 435 W-F 555 W)$. The individual color excesses and extinctions of the member MS stars are listed in Table 3. Table 4 presents the corresponding mean values for the whole sample.

Within the limitations of our photometry, we cannot estimate the amount of multiplicity among the member stars. However, the optical color of a star hotter than B7 $\left(\sim 4 \mathrm{M}_{\odot}\right)$ will not be significantly affected by an equal-mass or less massive companion. The mean reddening inside the cluster, $E(F 435 W-F 555 W)=1.33 \pm 0.12 \mathrm{mag}$ (Table $4)$, agrees well with the result of Sung \& Bessell $(2004, E(B-V)=1.25-1.4$ mag for stars within $0.7 \mathrm{pc}$ from the center), who derived the reddening by ZAMS fitting to the CMD. We plot the $E(F 435 W-F 555 W)$ of the member MS stars as a function of their cluster-centric distances in panel a of Figure 6. The error bars indicate the uncertainty of the $E(F 435 W-F 555 W)$ values. There is an apparent trend of increasing $E(F 435 W-$ $F 555 \mathrm{~W}$ ) values (red dots) with cluster-centric radius, implying that differential reddening even exists within the cluster (of $r \leq 0.7 \mathrm{pc}$ ), just as was found earlier by Sung \& Bessell (2004; Figure 5 in their paper).

\section{Total to selective extinction ratio}

The mean ratio of total to selective extinction inside the cluster is $R_{F 555 W}=3.54 \pm 0.63$ (see Table 4) computed from the extinction $A_{F 555 W}$ and the color excess $E(F 435 W-F 555 W)$. The
$R_{F 555 W}$ value of majority of the cluster member stars exceeds 3.1 (Table 3, see Figure 7).

We adopt the $E(B-V)$ value of $1.1 \mathrm{mag}$ obtained by Pandey et al. (2000) as an upper limit for the foreground reddening towards the HD 97950 cluster. After we correct for foreground reddening (assuming Fitzpatrick 1999 with $R_{V}=3.1$ ), the mean $R_{F 555 W}$ increases to $R_{F 555 W}=3.75 \pm 0.87$. In Section 5.3, we have shown that the spectral type uncertainty in our selected five MS member stars (see Table 2) does not significantly affect the final $R_{V}$ value.

To investigate the spatial dependence of dust properties in HD 97950, we plot $R_{F 555 W}$ and $A_{F 555 W}$ as a function of cluster-centric distance in panels b and $\mathrm{c}$ of Figure 6, both of which show significant scatter. We computed the average values of $R_{F 555 W}$ and $A_{F 555 W}$ within concentric annuli, respectively, with an initial radius of $0.1 \mathrm{pc}$ increasing to $0.5 \mathrm{pc}$ in steps of $0.1 \mathrm{pc}$. The mean values are plotted as red dots in panels $\mathrm{b}$ and $\mathrm{c}$ of Figure 6 . The mean $R_{F 555 W}$ and $A_{F 555 W}$ values remain roughly constant within the uncertainties, showing a possible slight increase in the outermost annulus.

The dispersion of $R_{F 555 W}, \sigma_{R_{F 555 W}}$, is larger in the innermost region of the cluster than in the outer part (panel c). The large variation of $A_{F 555 W}$ and $R_{F 555 W}$ inside the cluster may imply that the column density of dust within the cluster HD 97950 and possibly the size of dust grains vary along different lines of sight, resulting in a clumpy dust distribution across the cluster. This could be the result of stellar feedback from massive stars, which is destroying dust grains and modifying their distribution.

\section{Discussion}

\subsection{Inferred dust growth history for the HD 97950 region}

A high value of $R_{V}$ (>3.1) likely indicates that the size of dust grains in the HD 97950 cluster is larger than the mean grain size in the diffuse ISM. The growth of dust grains, which may have occurred via accretion or coagulation (Hirashita 2012), took place in the parental molecular cloud of the cluster, where the dust grains can accrete mantles of molecules (e.g., ice) or elements (e.g., $\mathrm{C}, \mathrm{N}$, and $\mathrm{O}$ ) from the gas phase (Cardelli et al. 
1989). Accretion of volatile ice mantles on the grains increases the sticking coefficients in graingrain collisions (Ossenkopf 1993), hence enhancing the chance of coagulation, i.e., the adherence of dust particles driven by their mutual interaction. Though a higher cloud density promotes coagulation (Tielens 1989), the latter depends also on the particles' collision velocity, which is a function of mass, elastic properties, and surface energy of the dust material (Ossenkopf 1993). Cardelli et al. (1989) computed the integrated extinction cross sections in several dense Galactic regions $\left(R_{V}>3.1\right)$, and found that their extinction cross section is smaller than that of the diffuse ISM. Since accretion increases the total extinction cross section while coagulation either increases or decreases it, they suggested that the size growth of grains is dominated by coagulation, which is confirmed by a recent study of Campeggio et al. (2007).

Whittet et al. (2001) found that when dust grows via coagulation, $R_{V}$ increases on average with $A_{V}$ across the whole extinction range, while mantle growth (accretion) requires a threshold extinction below which $R_{V}$ is independent of $A_{V}$. In the cluster HD 97950, the trend that $R_{F 555 W}$ increases almost linearly with $A_{F 555 W}$ (panel d in Figure 6) implies that large dust grains might have formed via coagulation in the parental cloud of this cluster.

\subsection{Extinction Curve within HD 97950}

We have obtained extinctions in the F220W, F250W, F330W, F435W, F555W, F675W, and $\mathrm{F} 814 \mathrm{~W}$ filters for the cluster MS member stars (Table 3) in Section 3. We adopt the foreground reddening from Pandey et al. (2000), with an assumption of a Fitzpatrick (1999) extinction law for the foreground $\left(R_{V}=3.1\right)$. In Figure 8, we compare the mean extinction curves (corrected for foreground reddening) $E(\lambda-$ $F 555 W) / E(F 435 W-F 555 W)$ (left panel) and $A_{\lambda} / A_{F 555 W}$ (right panel) for the $111 \mathrm{MS}$ members (black line) and the five MS stars with spectroscopy (red line) from which we obtain the reddening vector of the cluster in Section 3. These two extinction curves agree quite well with each other, confirming the consistency of our method.

In Figure 9, we plot the mean extinction curves for the MS members (black line) already corrected for the foreground extinction, and compare it with Cardelli et al.'s $\left(R_{V}=3.1,1989\right)$, Fitzpatrick's $\left(R_{V}=3.1,1999\right)$, and Calzetti et al.'s $\left(R_{V}=4.05\right.$, 2000 ) extinction laws (red dashed lines). We normalize the extinction curves to the visual color excess $E(F 435 W-F 555 W)$ (upper panels) and extinction $A_{\lambda} / A_{F 555 W}$ (lower panels). As the $R_{V}$ of the HD 97950 cluster is larger than the mean Galactic value 3.1, we also plot the resulting extinction laws of Fitzpatrick (1999), Cardelli et al. (1989) and Calzetti et al. (2000) using the $R_{V}$ value of the cluster, 3.75, for comparison (red solid lines). Calzetti et al.'s (2000) extinction law is independent of $R_{V}$ when it is expressed as $E(\lambda-F 555 W) / E(F 435 W-F 555 W)$. We indicate the uncertainties of Fitzpatrick's (1999) and Calzetti et al.'s (2000) extinction laws with error bars. Uncertainties are not provided in the study of Cardelli et al. (1989).

At optical wavelengths (filter passbands $F 814 \mathrm{~W}$ to $F 435 \mathrm{~W}$ ) all three extinction law prescriptions are essentially indistinguishable from our data. In the mid-UV, within the standard deviation associated with $E(\lambda-F 555 W) / E(F 435 W-F 555 W)$ (error bars), the extinction curve (black line) we obtained for the HD 97950 cluster (upper panels) tends to be greyer than the Galactic extinction laws with $R_{V}=3.75$ (red dotted lines) and $R_{V}=3.1$ (red dashed lines, Cardelli et al. 1989; Fitzpatrick 1999). The cluster extinction curve is closer to the extinction law of Calzetti et al. (2000), especially in the F220W (MUV) pass bands (black box). However, we cannot distinguish the cluster extinction curve normalized to the visual extinction $A_{\lambda} / A_{F 555 W}$, from the laws of Fitzpatrick (1999), Cardelli et al. (1989) and Calzetti et al. (2000) with the same $R_{V}=3.75$ as the cluster (lower panels). Since a possible age spread, unrecognized binaries, and errors in assigning spectral types all may contribute to systematic errors in the absolute magnitude measurements while colors are less affected, plotting $E(\lambda-F 555 W) / E(F 435 W-F 555 W)$ is more sensitive for detecting potential differences between extinction curves.

We have assumed that for the foreground extinction the standard $R_{V}$ value holds, i.e., $R_{V_{\text {fore }}}=3.1$. In order to explore how a different ratio of total to selective extinction would affect the resulting cluster extinction curve, we 
test other values. Choosing a smaller $R_{V_{\text {fore }}}$ of 2.6 makes the cluster extinction curve greyer, whereas it stays almost unchanged when increasing $R_{V_{\text {fore }}}$ to 3.6 .

Both the extinction laws of Cardelli et al. (1989) and Fitzpatrick (1999) for $R_{V}$ values of 3.1 and 3.75 show a prominent UV bump at $0.2175 \mu \mathrm{m}$ (Figure $1 \&$ 9). However, the mean extinction curve of the HD 97950 cluster is similar to the grey extinction law of starburst galaxies (Calzetti et al. 2000), which is characterized by the absence of the $0.2175 \mu \mathrm{m}$ bump in the midUV (F220W, Figure $1 \& 9)$. To investigate the strength of the UV bump, we derive $E(F 220 W-$ $F 555 W) / E(F 435 W-F 555 W)$ from the extinction laws of Fitzpatrick (1999) and Cardelli et al. (1989) computed with the individual $R_{F 555 W}$ values of all MS member stars (Table 3$)$. The $E(F 220 W-F 555 W) / E(F 435 W-F 555 W)$ values of the member stars are all smaller than those derived from Fitzpatrick's (1999, red line) and Cardell et al.'s (1989, black line) law, except for five stars (Figure 10). This points to a greyer extinction in the HD 97950 cluster than the Galactic average one.

Studies of Pierini et al. (2005) and Panuzzo et al. (2007) both found that the strength of the UV bump decreases when dust is mostly composed of large grains that formed in a very dense molecular cloud and absorb less UV radiation with respect to smaller grains (Hirashita 2012). As shown by Witt \& Gordon (2000) in the case of the Milky Way, a clumpy dust distribution can lead to the greyness of the extinction curve and the presence of a reduced UV bump. Our measurement of the color excess and extinction of stars in the HD 97950 cluster indicates that the dust distribution within this cluster is likely clumpy (see Section 4).

\subsection{Individual Extinction Curve of mem- ber stars}

As a consistency check, we plot in Figure 11 the individual extinction curves of the five MS spectroscopic member stars, from whose spectral types we could derive independent color excesses and absolute extinctions. We compare the spectral types determined by Melena et al. (2008) and those by other studies (Moffat 1983, Drissen et al. 1995), and find a typical difference of two spectral sub-classes. Therefore, we assume that the uncer- tainty on the spectral type of these five stars to be two sub-classes, which corresponds to an uncertainty in the range of $\sim 0.002-0.02$ on the $E(\lambda-F 555 W) / E(F 435 W-F 555 W)$ (the size of the filled circles in Figure 11) of these five stars.

The $R_{V}$ values of these stars span the range 2.48-4.06, and their visual extinctions range from 3.29 to $5.0 \mathrm{mag}$. Their extinction curves (in red) are compared with the extinction laws of Cardelli et al. (1989), Fitzpatrick (1999) and Calzetti et al. (2000) computed for their original $R_{V}=3.1$ and 4.05 (blue dashed lines), for the stars' measured $R_{V}$ as indicated in the upper panels (blue dotted lines), and the extinction laws of Cardelli et al. (1989) and Fitzpatrick's (1999) extinction laws with $R_{V}$ values from 2.0 to 6.0 (grey shaded regions), which is the range of $R_{V}$ values for the stars found when deriving Galactic extinction laws in Fitzpatrick's and Cardelli et al's papers. The typical feature of these extinction laws is the existence of the bump at $0.2175 \mu \mathrm{m}$ for all $R_{V}$ values.

As shown in Figure 11, the extinction curves of these five stars with known spectral types appear greyer than the extinction law of Fitzpatrick (1999) for the observed $R_{V}$ values (2.0-6.0), and greyer than Cardelli et al.'s (1989) law when calculated with the stars' $R_{V}$ values. Though Cardelli et al. (1989) law with $R_{V} \sim 5.0$ can reproduce the individual extinction value for the values in the wavelength of F220 W, its values at the wavelengths of $F 250 \mathrm{~W}$ and $F 330 \mathrm{~W}$ filters are below the observed values of the five MS stars, resembling the feature of an UV bump. The absence of an UV bump in the individual extinction curve closely mimicks the extinction law of Calzetti et al. (2000) independently of $R_{V}$. The same can be seen when comparing the extinction laws that were normalized to the visual extinction $A_{F 555 W}$. These results point to the clumpiness of the dust distribution as the main cause for the greyness of the stars' extinction curves.

\section{Summary}

We use the F220W, F250W, F330W, F435W, $F 555 \mathrm{~W}, \mathrm{~F} 675 \mathrm{~W}$, and $F 814 \mathrm{~W}$ photometry obtained with HST HRC/ACS and WFPC2 to estimate individual stellar reddenings and extinctions for stars in the HD 97950 cluster. The main results of our analysis are: 
We derive stellar reddenings for 111 individual MS member stars inside the HD 97950 cluster. The mean line-of-sight reddening inside the cluster is $E(F 435 W-F 555 W)=1.33 \pm 0.12 \mathrm{mag}$ (with $R_{F 555 W}=3.54 \pm 0.63$, without foreground reddening correction). Using only the value that is corrected for foreground extinction, the total to selective extinction ratio is $R_{F 555 W}=3.75 \pm 0.87$. The large dispersion of $R_{F 555 W}$ indicates a likely clumpy distribution of dust within the cluster.

Within the standard deviation associated with $E(\lambda-F 555 W) / E(F 435 W-F 555 W)$ in each filter, the extinction curve of the HD 97950 cluster in the filter F220 W corrected for foreground reddening tends to be greyer than the average Galactic extinction laws of Cardelli et al. (1989) and Fitzpatrick (1999) with both $R_{V}=3.75$ and $R_{V}=3.1$. The extinction curve is closer to the extinction law for starburst galaxies (Calzetti et al. 2000), showing a flatter extinction trend at the UV wavelengths. At longer wavelengths the cluster extinction curve agrees with all three extinction laws. This may indicate an anomalous extinction law in the HD 97950 cluster, and that the dust in the HD 97950 cluster has similar properties to the dust in starburst galaxies. The absence of the UV bump at $0.2175 \mu \mathrm{m}$ for the cluster and starburst galaxies may be attributed to the clumpy dust distribution, and, in addition, to dust grains that grew larger than those in the average Galactic ISM because of the high density of the cluster parental molecular cloud (Pierini et al. 2005; Panuzzo et al. 2007).

We thank Matthias Frank for his Python script to derive the offsets between ACS images. We are also grateful to the referee for his/her indepth comments, which improved this paper. This work is funded by National Natural Science Foundation of China, No: 11503015, and the fund of Shanghai education committee, No: 1021ZK151009027-ZZyy15104. XYP acknowledges travel support by Sonderforschungsbereich 881 "The Milky Way System" of the German Research Foundation (subproject B5). XYP is a member of the Silk Road Project Team in National Astronomical Observatories of China (NAOC, http://silkroad.bao.ac.cn), and acknowledges the technical support from this team.

\section{REFERENCES}

Baraffe, I., Chabrier, G., \& Gallardo, J. 2009, ApJ, 702, L27

Calzetti, D., Kinney, A. L., \& Storchi-Bergmann, T. 1994, ApJ, 429, 582

Calzetti, D. 1997, AJ, 113, 162

Calzetti, D., Armus, L., Bohlin, R. C., et al. 2000, ApJ, 533, 682

Campeggio, L., Strafella, F., Maiolo, B., Elia, D., \& Aiello, S. 2007, ApJ, 668, 316

Cardelli, J. A., \& Savage, B. D. 1988, ApJ, 325, 864

Cardelli, J. A., Clayton, G. C., \& Mathis, J. S. 1989, ApJ, 345, 245

Chini, R., \& Krügel, E. 1983, A\&A, 117, 289

Chini, R., \& Wargau, W. F. 1990, A\&A, 227, 213

Clayton, C. A. 1986, MNRAS, 219, 895

Dolphin, A. E. 2000, PASP, 112, 1397

Dolphin, A.E. 2005, Dolphot User's Guide, http://purcell.as.arizona.edu/ Dolphot/

Dolphin, A.E. 2006, Dolphot/ACS User's Guide, http://purcell.as.arizona.edu/ Dolphot/

Draine, B. T. 2009, Cosmic Dust - Near and Far, ASP Conference Vol. 414, ed. Henning, T., Grün, E., \& Steinacker, J. (San Francisco: ASP), 453

Drissen, L., Moffat, A. F. J., Walborn, N. R., \& Shara, M. M. 1995, AJ, 110, 2235

Fernandes, B., Gregorio-Hetem, J., \& Hetem, A. 2012, A\&A, 541, A95

Fitzpatrick, E. L. 1999, PASP, 111, 63

Fitzpatrick, E. L., \& Massa, D. 2005, AJ, 130, 1111

Fitzpatrick, E. L., \& Massa, D. 2007, ApJ, 663, 320

Fitzpatrick, E. L., \& Massa, D. 2009, ApJ, 699, 1209 
Harayama, Y., Eisenhauer, F., \& Martins, F. 2008, ApJ, 675, 1319

Hirashita, H. 2012, MNRAS, 422, 1263

Kudryavtseva, N., Brandner, W., Gennaro, M., et al. 2012, ApJ, 750, L44

Larson, K. A. 2014, PASP, 126, 27

Lebouteiller, V., Brandl, B., Bernard-Salas, J., Devost, D., \& Houck, J. R. 2007, ApJ, 665, 390

Maclean, S., Duley, W. W., \& Millar, T. J. 1982, ApJ, 256, L61

Marigo, P., Girardi, L., Bressan, A., et al. 2008, A\&A, 482, 883

Massa, D., Savage, B. D., \& Fitzpatrick, E. L. 1983, ApJ, 266, 662

Mathis, J. S., Rumpl, W., \& Nordsieck, K. H. 1977, ApJ, 217, 425

Melena, N. W., Massey, P., Morrell, N. I., \& Zangari, A. M. 2008, AJ, 135, 878

Moffat, A. F. J. 1983, A\&A, 124, 273

Ossenkopf, V. 1993, A\&A, 280, 617

Pandey, A. K., Ogura, K., \& Sekiguchi, K. 2000, PASJ, 52, 847

Pang, X., Pasquali, A., \& Grebel, E. K. 2011, AJ, 142,132

Pang, X., Grebel, E. K., Allison, R. J., et al. 2013, ApJ, 764, 73

Panuzzo, P., Granato, G. L., Buat, V., et al. 2007, MNRAS, 375, 640

Pierini, D., Maraston, C., Gordon, K. D., \& Witt, A. N. 2005, MNRAS, 363, 131

Savage, B. D., Massa, D., Meade, M., \& Wesselius, P. R. 1985, ApJS, 59, 397

Seab, C. G., \& Shull, J. M. 1983, ApJ, 275, 652

Seaton, M. J. 1979, MNRAS, 187, 73P

Sparke, L. S., \& Gallagher, J. S., 2007, Galaxies in the Universe, Cambridge, UK: Cambridge University Press
Sung, H., \& Bessell, M. S. 2004, AJ, 111, 1014

Tielens, A. G. G. M., 1989. In Allamandola, L., Tielens, A. G. G. M., editors, Interstellar Dust, IAU Symp. 135, page 239, Kluwer Academics Publisher, Dordrecht

Valencic, L. A., Clayton, G. C., \& Gordon, K. D. 2004, ApJ, 616, 912

Weingartner, J. C., \& Draine, B. T. 2001, ApJ, 548, 296

Whittet, D. C. B., Gerakines, P. A., Hough, J. H., \& Shenoy, S. S. 2001, ApJ, 547, 872

Witt, A. N., \& Gordon, K. D. 2000, ApJ, 528, 799

Wood, K., Whitney, B. A., Robitaille, T., \& Draine, B. T. 2008, ApJ, 688, 1118

Yadav, R. K. S., \& Sagar, R. 2001, MNRAS, 328, 370

This 2-column preprint was prepared with the AAS LATEX macros v5.2. 


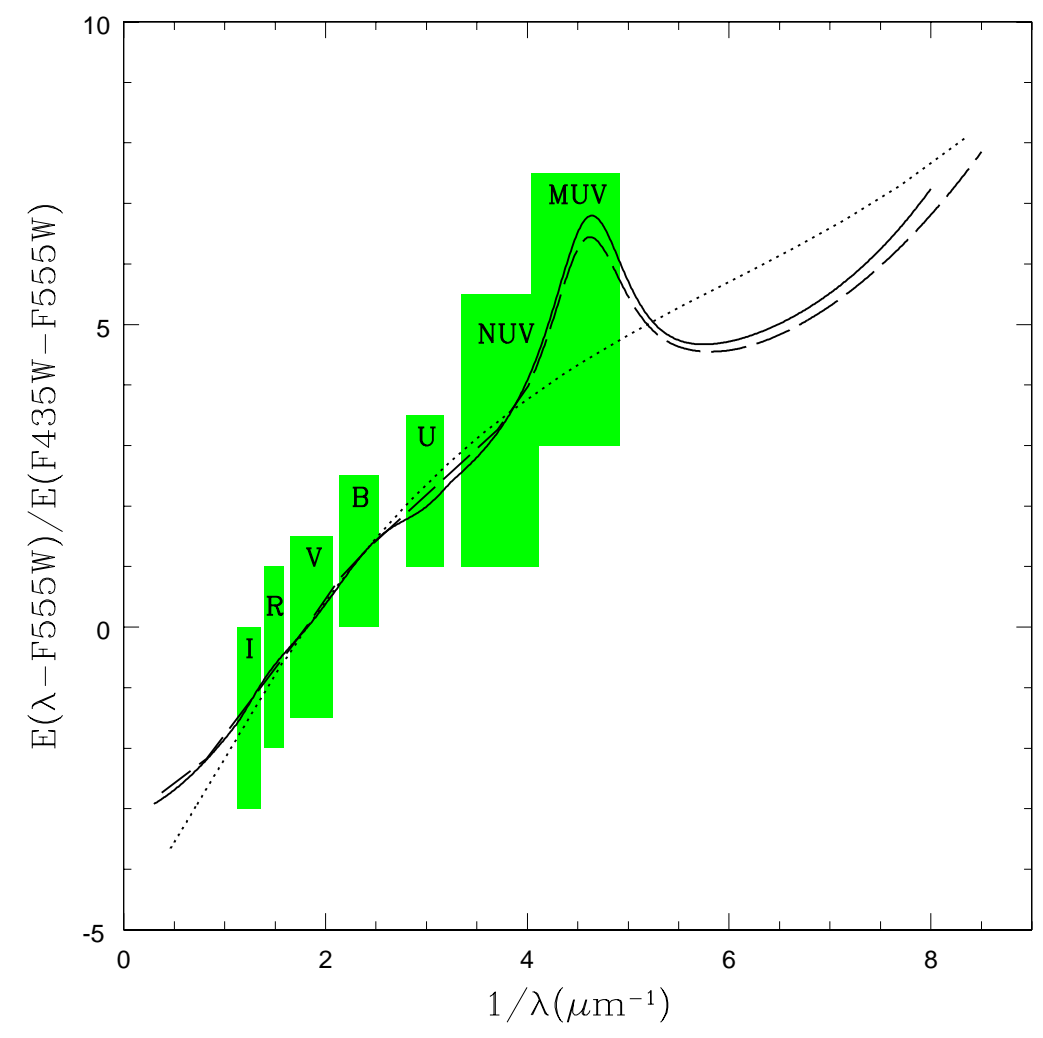

Fig. 1.- Average Galactic extinction curves $\left(R_{V}=3.1\right)$ of Cardelli et al. (1989, solid line) and Fitzpatrick (1999, dashed line). The dotted line is the mean extinction curve for 39 starburst galaxies (Calzetti et al. $2000)$ with $R_{V}=4.05$. The UV bump in the Galactic extinction curves is observed around $4.6 \mu \mathrm{m}^{-1}$. The shaded areas indicate the wavelength coverage of each filter. For convenience, we name the F220W, F250W, $F 330 W, F 435 \mathrm{~W}, F 555 \mathrm{~W}, \mathrm{~F} 675 \mathrm{~W}$, and $\mathrm{F} 814 \mathrm{~W}$ filters $M U V, N U V, U, B, V, R$, and $I$, respectively. 


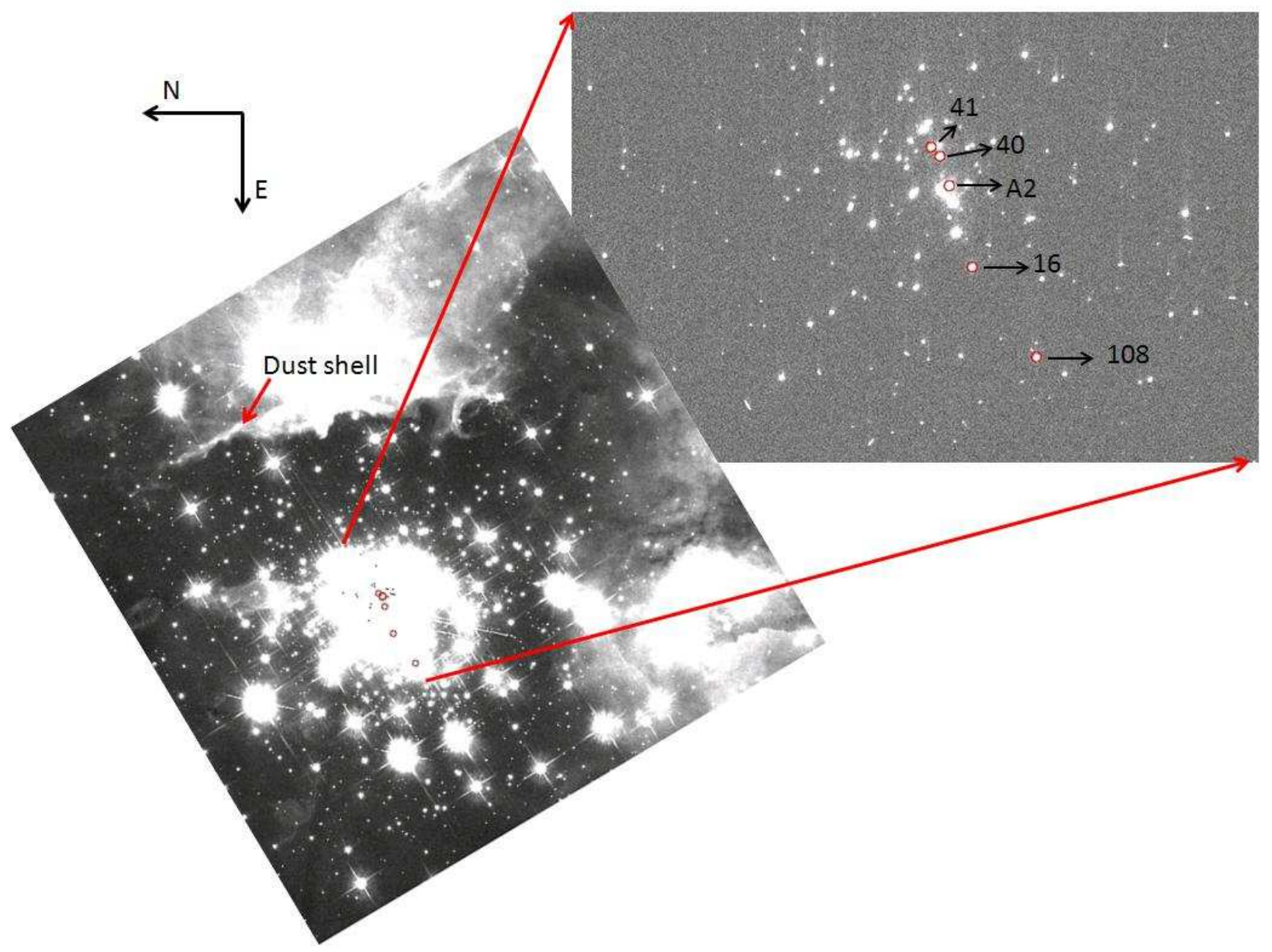

Fig. 2.- Image of the cluster HD97950 in the $F 555 \mathrm{~W}$ filter taken by the HST WFC3 UVIS (bottom left, $162^{\prime \prime} \times 162^{\prime \prime}$ ) and WFPC2 PC chip (upper right, $20^{\prime \prime} \times 20^{\prime \prime}$ ). The WFPC2 PC image is a zoomed-in version of the cluster center which is saturated in the WFC3 image. The five MS stars with spectral types available from Melena et al. (2008) are indicated with red circles and their designations given by Melena et al. (2008). The dust shell, which is about $1.2 \mathrm{pc}$ away from the center, is indicated by an arrow. 


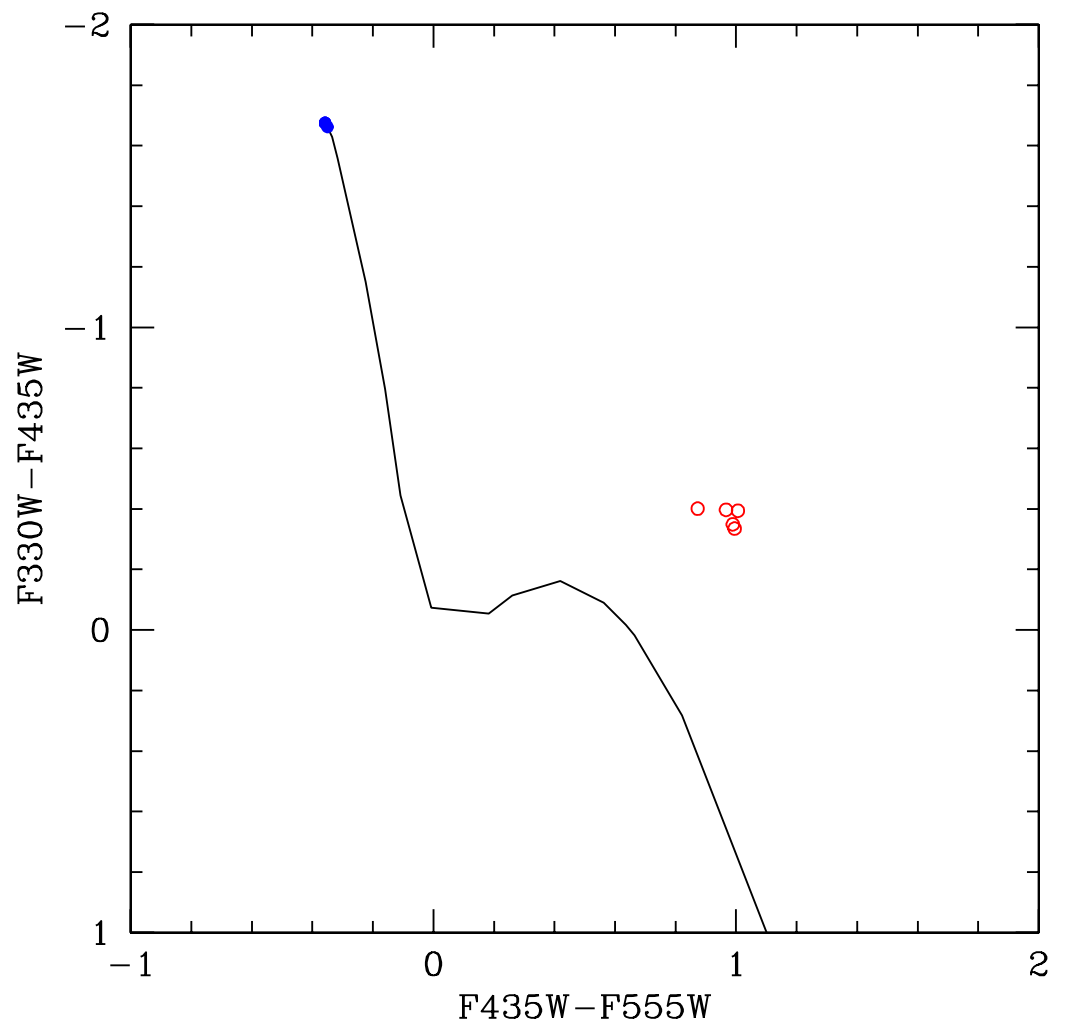

Fig. 3.- Color-color diagram of the five MS member stars whose spectral types are available from Melena et al. (2008). The black curve is the empirical ZAMS from Sparke \& Gallagher (2007). The red open circles denote the observed colors of the five stars, while the blue filled circles indicate the intrinsic colors derived from their corresponding spectral type. 


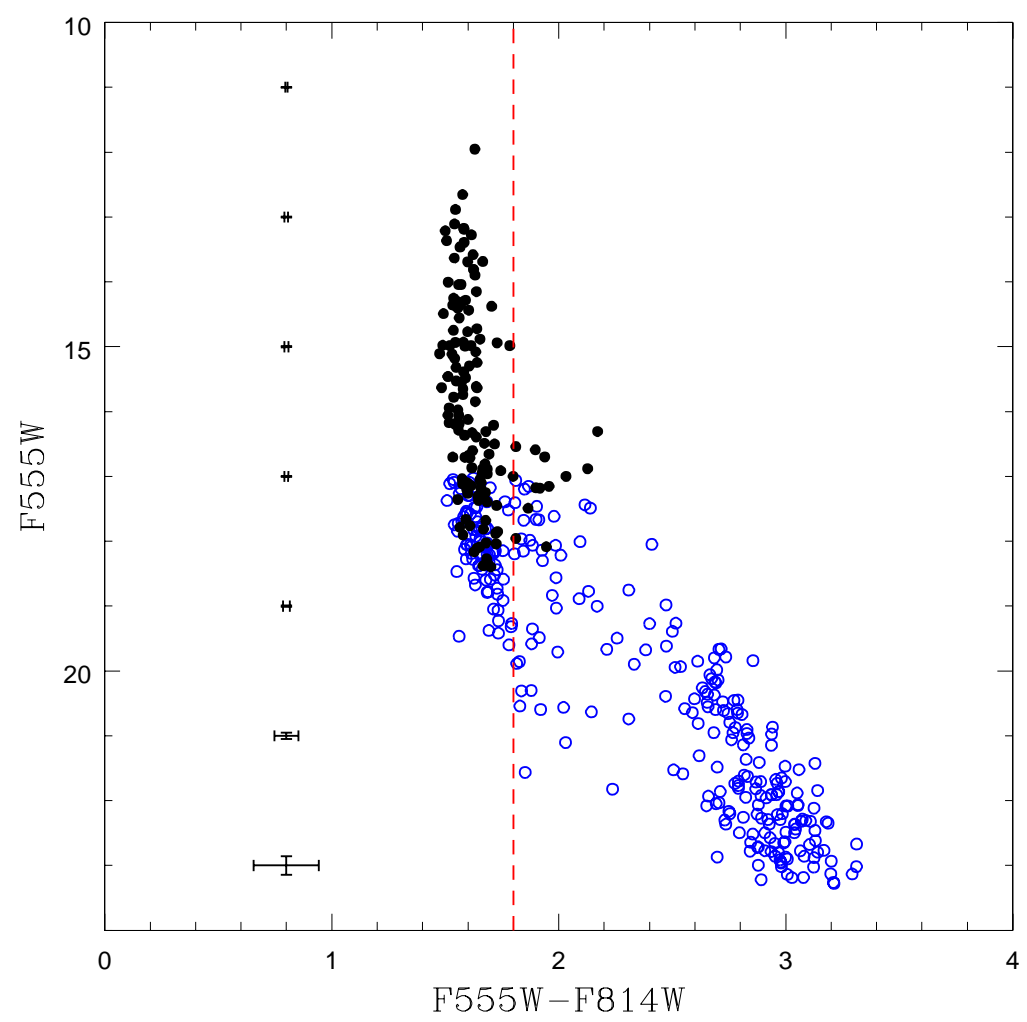

Fig. 4.- Color-magnitude diagram of all proper-motion-selected member stars with UV and optical photometry (black dots) and members fainter than $17 \mathrm{mag}$ in $F 555 \mathrm{~W}$ observed on the PC chip of the WFPC2 (blue open circles). Stars with UV-optical photometry that are bluer than $(F 555 W-F 814 W)=1.8$ mag (dashed vertical line) are selected as probable MS stars. 


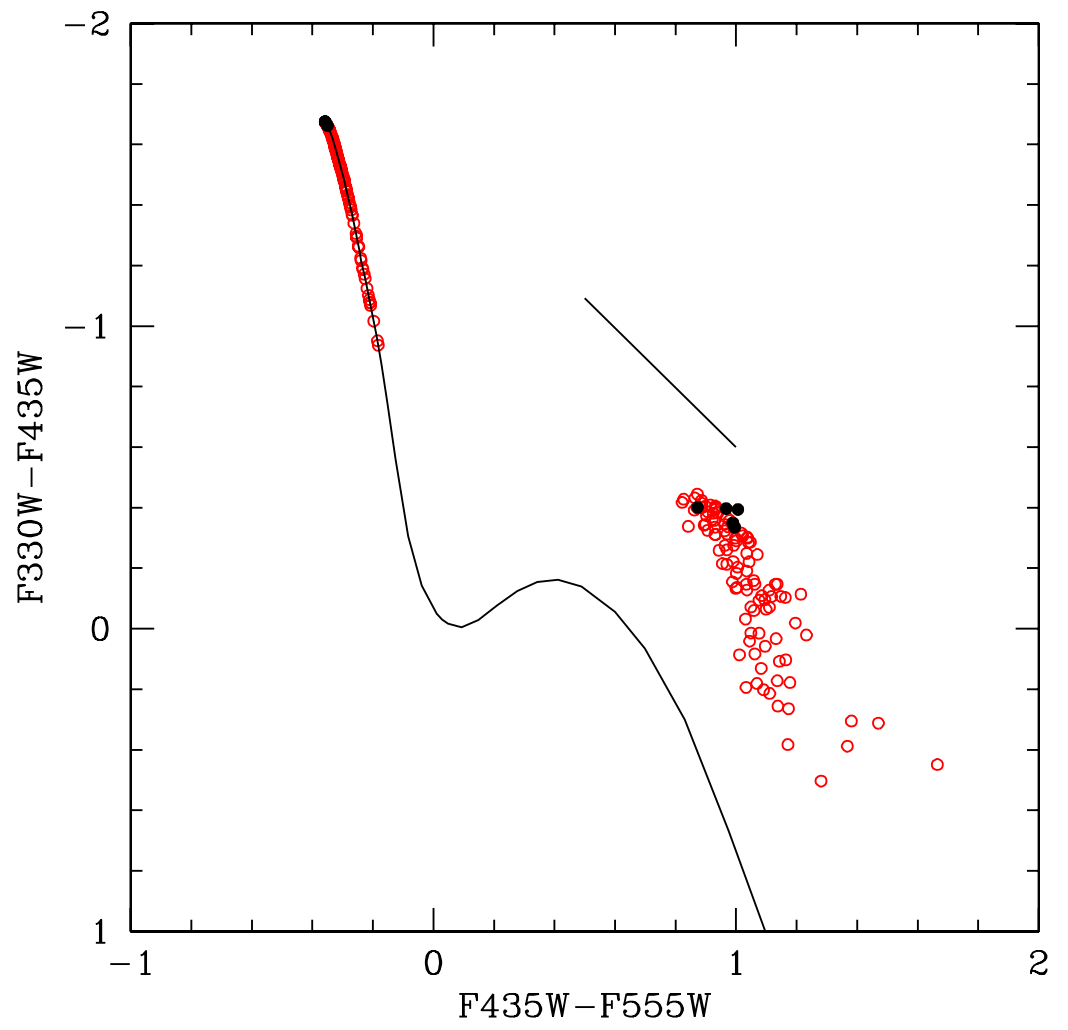

Fig. 5.- Color-color diagram of the selected MS member stars ( $F 555 W-F 814 W \leq 1.8$ mag). The black straight line indicates the slope of the reddening vector determined from the five MS member stars (black solid dots) whose spectral types are available from Melena et al. (2008). The MS member stars (red open dots) are projected onto the Marigo et al. (2008) isochrone (black curve) to obtain their reddening. 

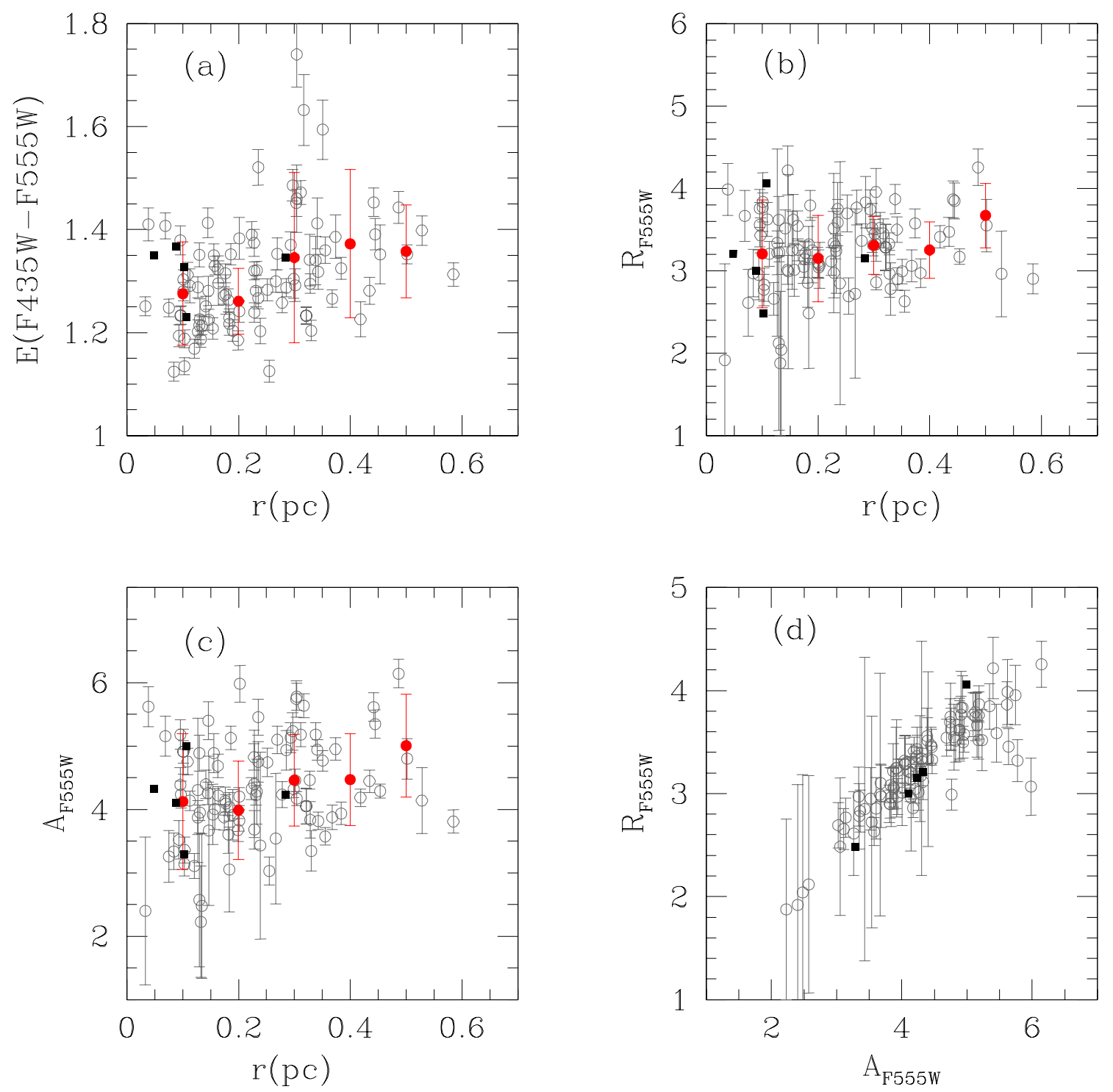

Fig. 6. - The dependence of the stellar reddening $E(F 435 W-F 555 W)$ (a), of the $R_{F 555 W}$ (b), and of the $A_{F 555 W}$ values (c) on the cluster-centric distance without foreground reddening correction, and the dependence of $R_{F 555 W}$ on $A_{F 555 W}$ (d). MS members for which no spectroscopy is available are plotted with grey open circles. The five MS member stars with spectral information from Melena et al. (2008) are indicated with filled black squares. In panel a, b, c, red filled circles and error bars represent the mean values and dispersions of $E(F 435 W-F 555 W), R_{F 555 W}$ and $A_{F 555 W}$ respectively. They were measured in concentric annuli around the cluster center. The innermost annulus was a circle with a radius of 0.1 pc. The next-larger annulus ranged from 0.1 to $0.2 \mathrm{pc}$ in the inner and outer radius, respectively, and for the subsequent annuli the radii were increased by $0.1 \mathrm{pc}$ each up to a maximum of $0.5 \mathrm{pc}$. 


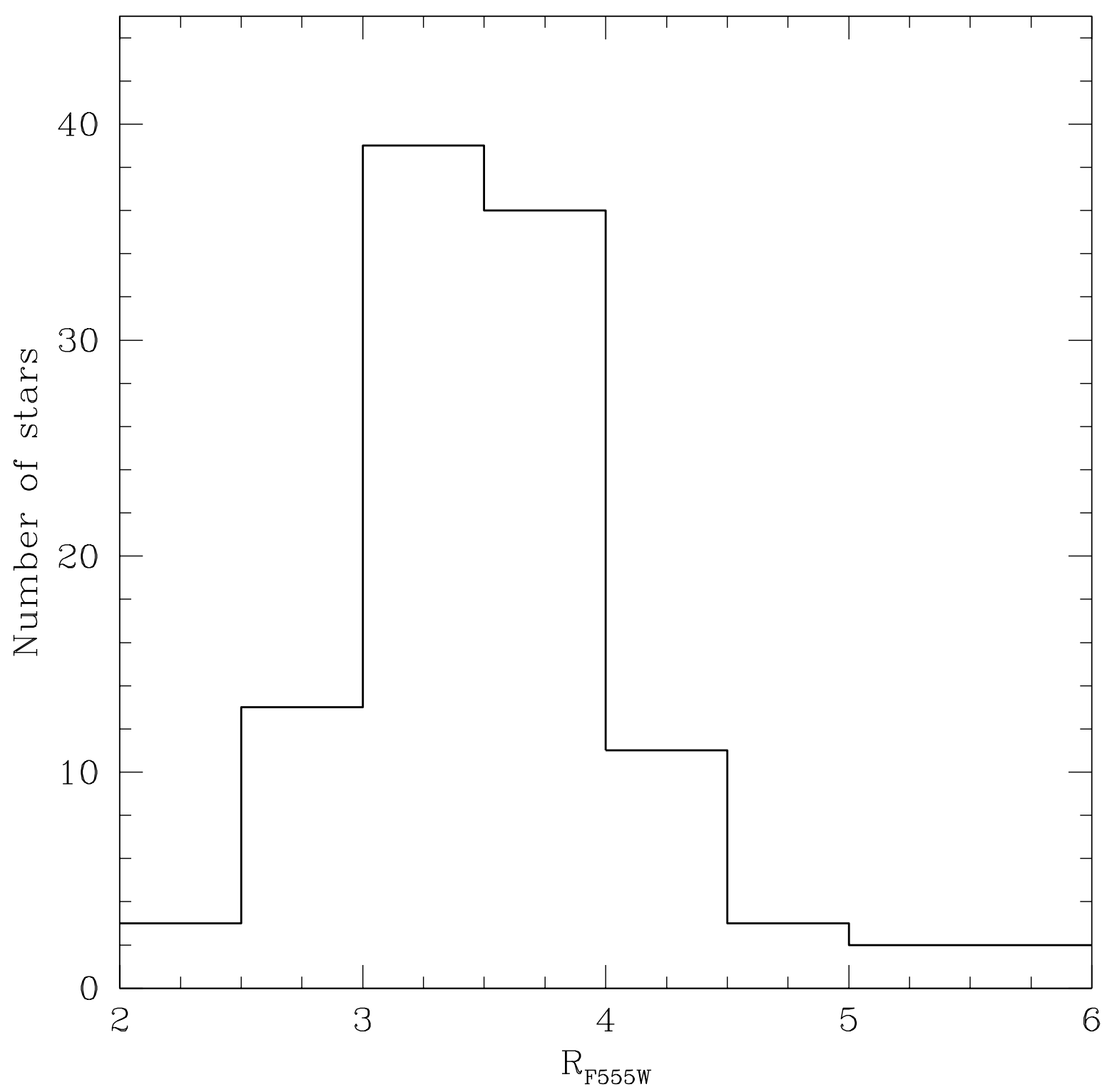

Fig. 7.- The histogram distribution of the $R_{F 555 W}$ values for all MS member stars (F555W$F 814 W \leq 1.8 \mathrm{mag})$. 

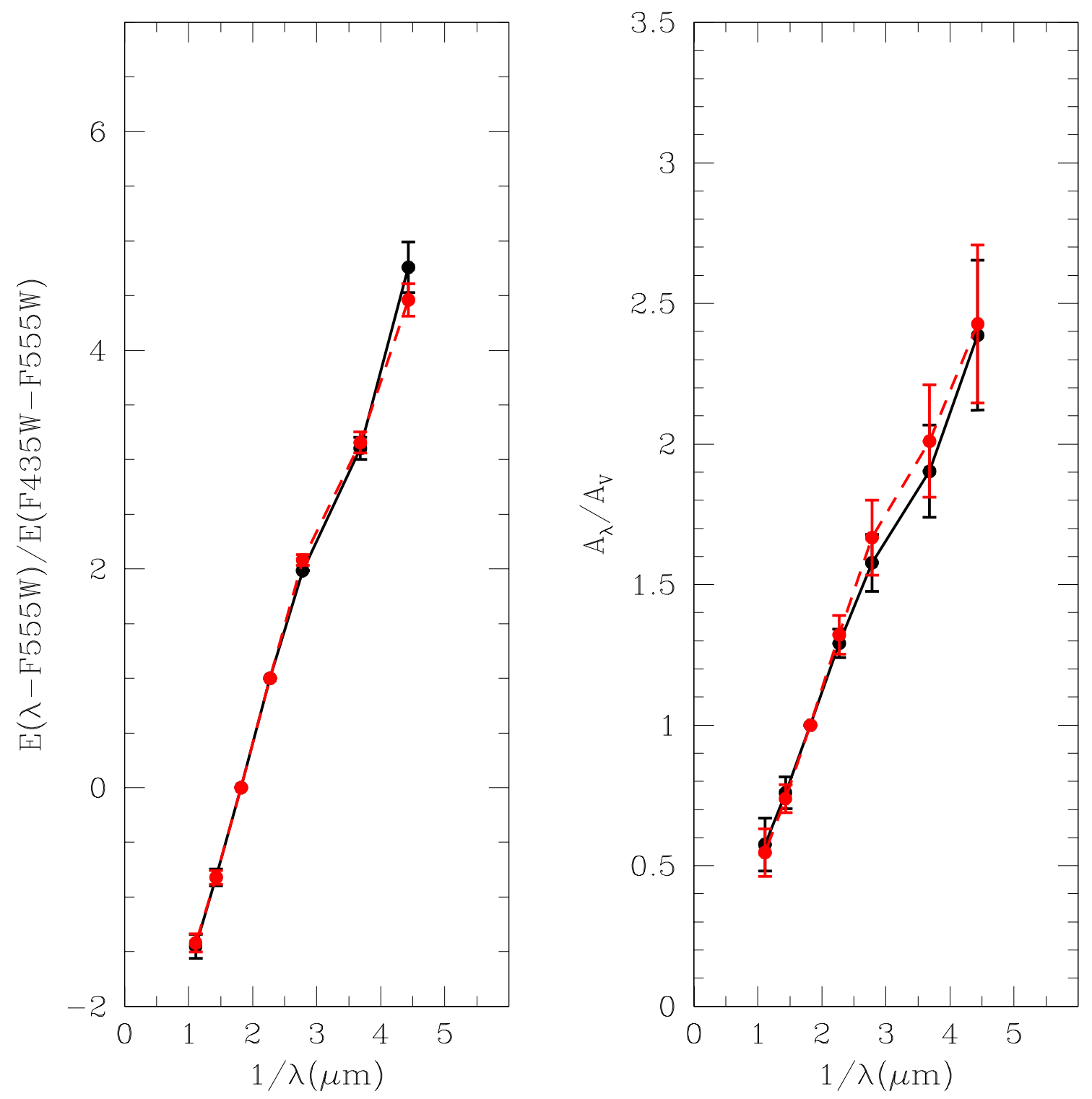

Fig. 8. - Mean extinction curve $E(\lambda-F 555 W) / E(F 435 W-F 555 W)$ (left panel) and $A_{\lambda} / A_{F 555 W}$ (right panel) already corrected for foreground extinction for the HD 97950 cluster (black lines, mean extinctions of the selected MS member stars) and the five main-sequence member stars with spectroscopy (red dashed lines, Melena et al. 2008) from which the cluster reddening vector is derived (Section 3). The error bars are the standard deviation associated with $E(\lambda-F 555 W) / E(F 435 W-F 555 W)$ and $A_{\lambda} / A_{F 555 W}$ in each filter. 

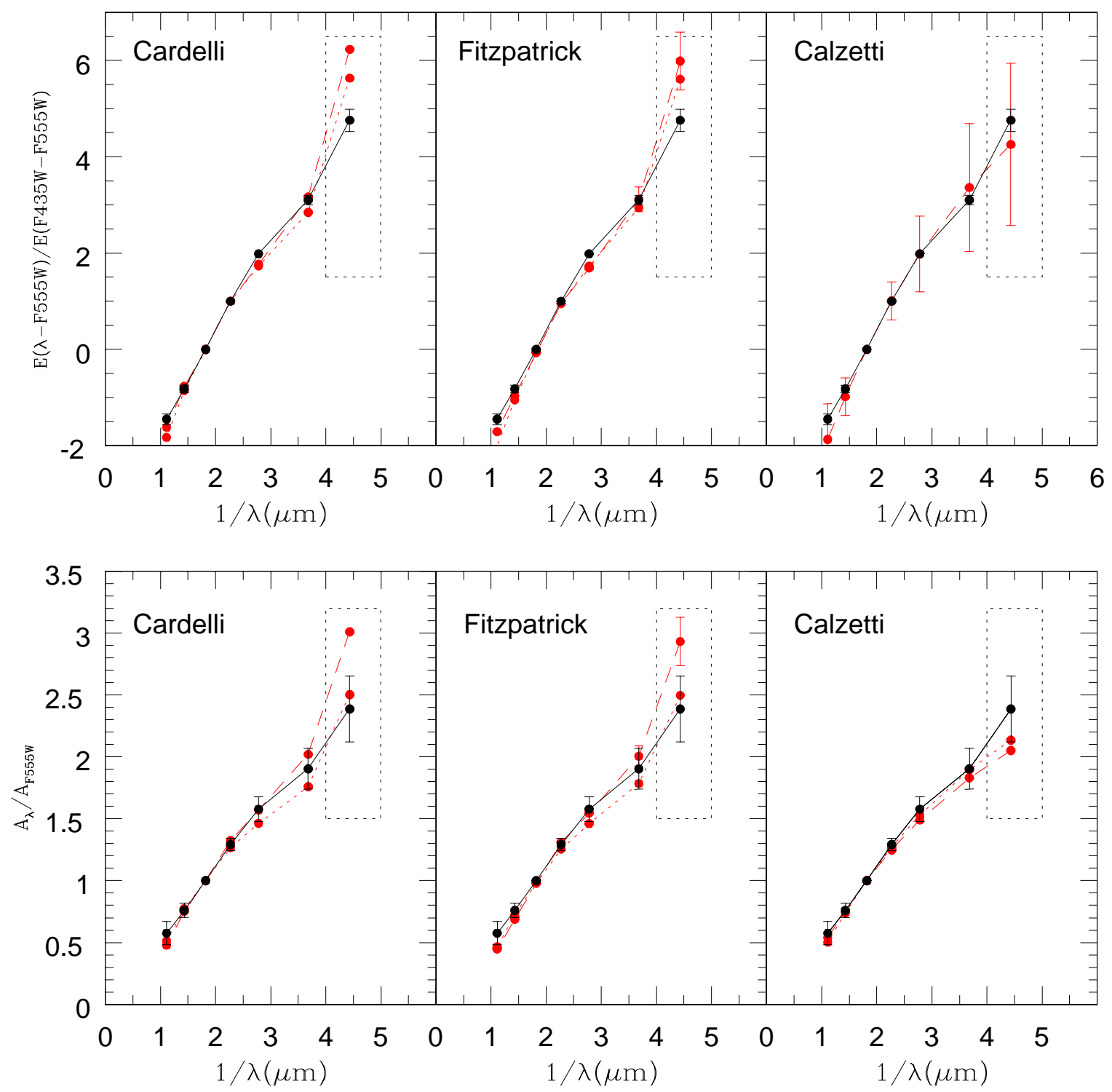

Fig. 9.- Mean extinction curve $E(\lambda-F 555 W) / E(F 435 W-F 555 W)$ (upper panels) and $A_{\lambda} / A_{F 555 W}$ (lower panels) for the HD 97950 cluster (black solid lines) already corrected for foreground extinction. The red dotted lines trace Cardelli et al.'s (1989), Fitzpatrick's (1999) and Calzetti et al.'s (2000) extinction laws with the de-reddened value of $R_{V}$ as the cluster HD $97950\left(R_{V}=3.75\right)$. The red dashed lines are the average Galactic extinction laws $\left(R_{V}=3.1\right)$ of Cardelli et al. (1989) and Fitzpatrick (1999), and Calzetti et al.'s (2000) extinction law for starburst galaxies $\left(R_{V}=4.05\right)$. The error bars are the standard deviation associated with $E(\lambda-F 555 W) / E(F 435 W-F 555 W)$ and $A_{\lambda} / A_{F 555 W}$ in each filter. Uncertainties of Fitzpatrick's (1999) and Calzetti et al.'s (2000) extinction laws are also indicated with error bars. For Fitzpatrick's (1999) extinction law, its uncertainty approaches zero for wavelengths larger than $4000 \AA$ owing to the normalization to the visual band. No such uncertainty information is available for Cardelli et al.'s (1989) extinction law. The dotted black boxes cover the $M U V$ filter where the cluster extinction curve differs most dramatically from the reference extinction laws. 


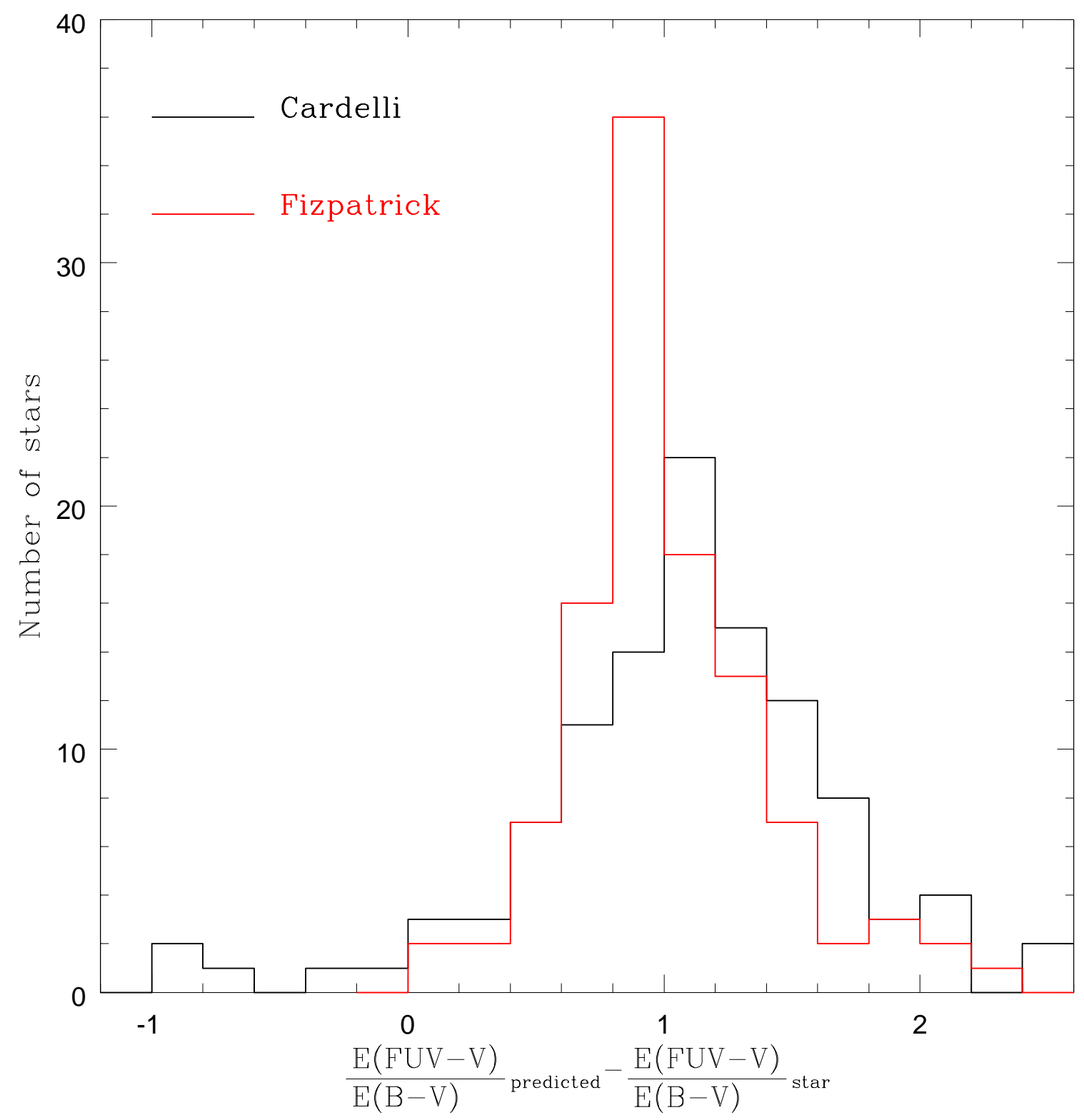

Fig. 10. - The histogram distribution of the difference of the predicted reddening ratio of $E(F U V-$ $V) / E(B-V)$ obtained for the Cardelli et al. (1989, black line) and the Fitzpatrick (1999, red line) extinction laws and the inferred extinction ratios in the corresponding $E(F 220 W-F 555 W) / E(F 435 W-F 555 W)$ values for the individual member stars in the HD 97950 cluster. For the calculation of each difference the $R_{F 555 W}$ derived for the individual star was adopted. A difference of positive value indicates a reduced or a missing of UV bump for the individual member star. 


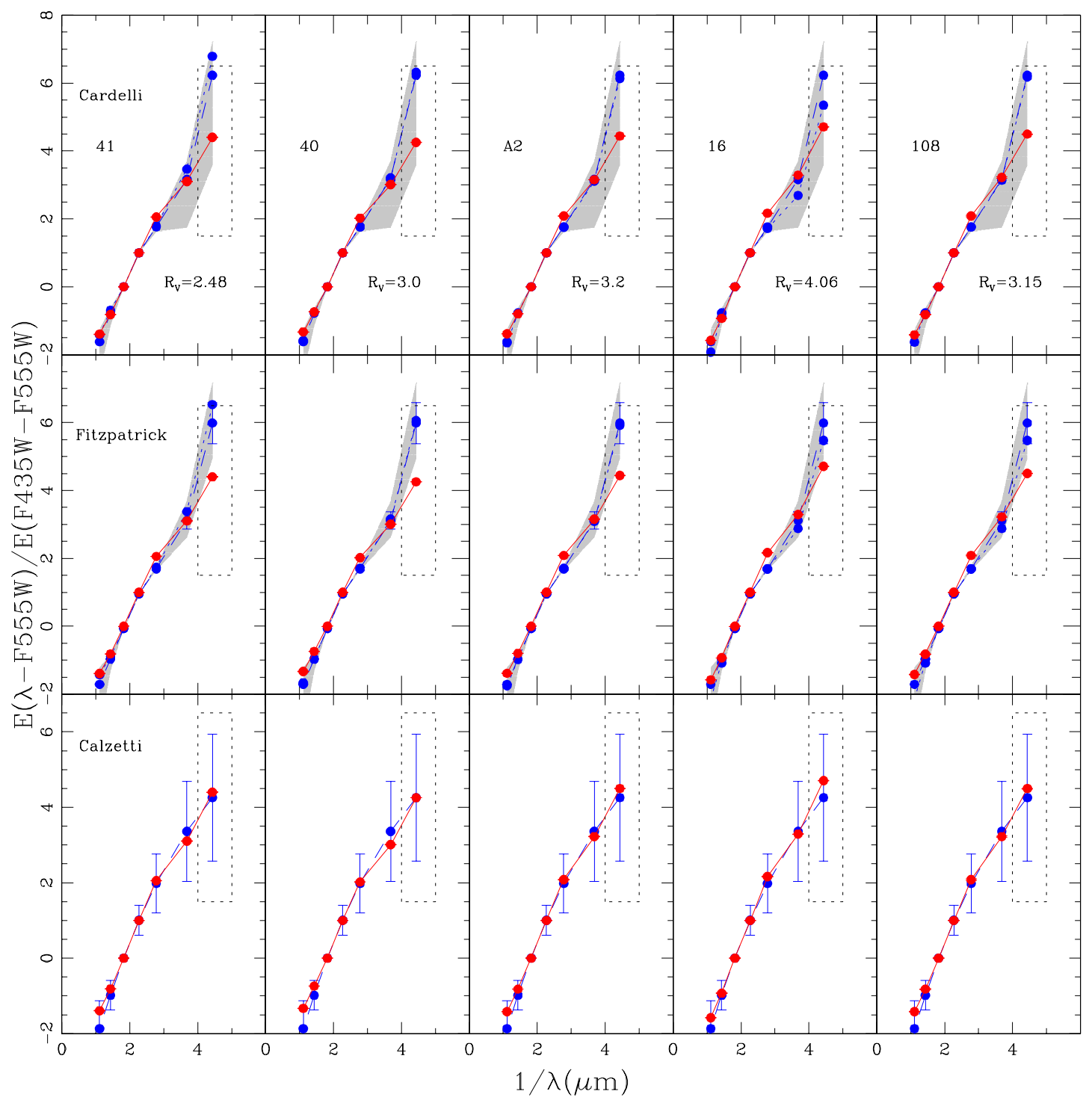

Fig. 11.- Individual extinction curves $E(\lambda-F 555 W) / E(F 435 W-F 555 W)$ for the five MS member stars with available spectroscopy from Melena et al. (2008) (red lines) already corrected for foreground extinction (panels of the same column). The error bar of $E(\lambda-F 555 W) / E(F 435 W-F 555 W)$ in each filter is of the size of data points. The designations of the stars as given by Melena et al. (2008) are indicated in the upper panels. The blue dashed lines (panels of the same row) are the average Galactic extinction laws $\left(R_{V}=3.1\right)$ of Cardelli et al. (1989) and Fitzpatrick (1999), and Calzetti et al.'s (2000) extinction law for starburst galaxies $\left(R_{V}=4.05\right)$. The blue dotted lines trace Cardelli et al.'s (1989) and Fitzpatrick's (1999) laws with the same value of $R_{V}$ as for each individual star (indicated in the upper panels). The grey shaded regions are Cardelli et al.'s (1989) and Fitzpatrick's (1999) laws with $R_{V}$ between 2.0 (upper boundary) and 6.0 (lower boundary). The meaning of the error bars and the dotted black box are the same as in Figure 9. 
Table 1: The UV and optical photometry of member stars in the HD 97950 cluster

\begin{tabular}{|c|c|c|c|c|c|c|c|c|c|c|c|c|c|c|c|c|}
\hline No. & R.A. & $D e c$ & F220W & $\sigma_{220}$ & $F 250 \mathrm{~W}$ & $\sigma_{250}$ & F330W & $\sigma_{330}$ & $F 435 \mathrm{~W}$ & $\sigma_{435}$ & $F 555 \mathrm{~W}$ & $\sigma_{555}$ & $F 675 \mathrm{~W}$ & $\sigma_{675}$ & $F 814 W$ & $\sigma_{814}$ \\
\hline 1 & 168.7816010 & -61.2605972 & 15.34 & 0.003 & 13.51 & 0.002 & 12.64 & 0.002 & 12.99 & 0.002 & 11.95 & 0.003 & 11.00 & 0.003 & 10.32 & 0.023 \\
\hline 2 & 168.7804565 & -61.2608185 & 15.86 & 0.003 & 14.05 & 0.002 & 13.23 & 0.002 & 13.62 & 0.002 & 12.65 & 0.005 & 11.74 & 0.004 & 11.08 & 0.027 \\
\hline 3 & 168.7784424 & -61.2599373 & 16.00 & 0.003 & 14.24 & 0.003 & 13.42 & 0.003 & 13.85 & 0.003 & 12.89 & 0.005 & 12.00 & 0.005 & 11.34 & 0.030 \\
\hline 4 & 168.7788391 & -61.2608719 & 16.13 & 0.004 & 14.39 & 0.003 & 13.62 & 0.003 & 14.06 & 0.003 & 13.19 & 0.006 & 12.26 & 0.005 & 11.61 & 0.030 \\
\hline 5 & 168.7794037 & -61.2609558 & 16.26 & 0.004 & 14.48 & 0.003 & 13.66 & 0.003 & 14.08 & 0.003 & 13.11 & 0.005 & 12.22 & 0.005 & 11.57 & 0.028 \\
\hline 6 & 168.7803192 & -61.2597466 & 16.49 & 0.004 & 14.64 & 0.003 & 13.77 & 0.003 & 14.15 & 0.003 & 13.18 & 0.005 & 12.25 & 0.005 & 11.59 & 0.028 \\
\hline 7 & 168.7788544 & -61.2609329 & 16.40 & 0.004 & 14.65 & 0.003 & 13.86 & 0.003 & 14.28 & 0.003 & 13.39 & 0.006 & 12.45 & 0.005 & 11.81 & 0.028 \\
\hline 8 & 168.7759705 & -61.2602043 & 16.16 & 0.004 & 14.46 & 0.003 & 13.70 & 0.003 & 14.15 & 0.003 & 13.21 & 0.006 & 12.34 & 0.005 & 11.71 & 0.042 \\
\hline 9 & 168.7806244 & -61.2607231 & 16.48 & 0.004 & 14.68 & 0.004 & 13.82 & 0.004 & 14.20 & 0.003 & 13.28 & 0.006 & 12.36 & 0.007 & 11.66 & 0.040 \\
\hline 10 & 168.7788086 & -61.2602081 & 16.35 & 0.004 & 14.60 & 0.003 & 13.83 & 0.003 & 14.25 & 0.003 & 13.36 & 0.006 & 12.49 & 0.005 & 11.86 & 0.031 \\
\hline
\end{tabular}

Note.- - Table 1 is presented in its entirety in the electronic edition of the Astronomical Journal. A portion is shown here for guidance regarding its form and content.

R.A. and Dec are Right Ascension and Declination of the member stars on the PC chip of WFPC2 based on the 2007 data. They are J2000 coordinates. The F220W, F250W, F330W, and F435W photometry is from ACS. The $F 555 W, F 675 W$, and $F 814 W$ photometry is from WFPC2 (2007 data). All magnitudes are Vega magnitudes in the ACS/HRC and WFPC2 filter systems.

Table 2: Photometry of main sequence stars with spectral types

\begin{tabular}{|c|c|c|c|c|c|c|c|c|c|c|c|c|}
\hline Designation & R.A. & $\overline{D e c}$ & F220W & F250W & F330W & $F 435 \mathrm{~W}$ & $F 555 \mathrm{~W}$ & $F 675 \mathrm{~W}$ & $F 814 W$ & Spectral type & $(U-B)_{0}$ & $(B-V)_{0}$ \\
\hline 41 & 168.7793427 & -61.2608604 & 17.50 & 15.70 & 14.91 & 15.31 & 14.44 & 13.50 & 12.83 & $\mathrm{O} 4 \mathrm{~V}$ & -1.210 & -0.320 \\
\hline 40 & 168.7796783 & -61.2608986 & 16.69 & 14.88 & 14.07 & 14.47 & 13.46 & 12.59 & 11.90 & O3V & -1.221 & -0.320 \\
\hline A2 & 168.7804565 & -61.2608185 & 15.86 & 14.05 & 13.23 & 13.62 & 12.66 & 11.74 & 11.08 & $\mathrm{O} 3 \mathrm{~V}$ & -1.221 & -0.320 \\
\hline 16 & 168.7825775 & -61.2605515 & 17.03 & 15.20 & 14.33 & 14.68 & 13.69 & 12.77 & 12.09 & O3V & -1.221 & -0.320 \\
\hline 108 & 168.7854614 & -61.2606812 & 17.33 & 15.50 & 14.56 & 14.89 & 13.90 & 12.96 & 12.27 & $05.5 \mathrm{~V}$ & -1.183 & -0.321 \\
\hline
\end{tabular}

Note.-All coordinates are J2000 coordinates. The colors $(U-B)_{0}$ and $(B-V)_{0}$ are taken from the empirical ZAMS of Sparke \& Gallagher $(2007)$. 
Table 3: The color excesses and extinctions of member stars

\begin{tabular}{|c|c|c|c|c|c|c|c|c|c|c|c|c|c|c|c|c|}
\hline No. & R.A. & $D e c$ & $E(M U V-B)$ & $E(N U V-B)$ & $E(U-B)$ & $E(B-V)$ & $E(V-R)$ & $E(V-I)$ & $A_{F 220 W}$ & $A_{F 250 W}$ & $A_{F 330 W}$ & $A_{F 435 W}$ & $A_{F 555 W}$ & $A_{F 675 W}$ & $A_{F 814 W}$ & $R_{F 555 \mathrm{~W}}$ \\
\hline 1 & 168.7788391 & -61.2608719 & 4.76 & 2.599 & 1.17 & 1.194 & 1.12 & 1.920 & 8.42 & 6.259 & 4.84 & 3.660 & 2.47 & 1.347 & 0.55 & 2.065 \\
\hline 2 & 168.7788544 & -61.2609329 & 4.82 & 2.639 & 1.20 & 1.220 & 1.12 & 1.920 & 8.86 & 6.677 & 5.24 & 4.038 & 2.82 & 1.694 & 0.90 & 2.310 \\
\hline 3 & 168.7806244 & -61.2607231 & 4.98 & 2.751 & 1.24 & 1.258 & 1.11 & 1.953 & 9.06 & 6.830 & 5.32 & 4.079 & 2.82 & 1.715 & 0.87 & 2.242 \\
\hline 4 & 168.7788086 & -61.2602081 & 4.81 & 2.636 & 1.20 & 1.222 & 1.06 & 1.844 & 9.24 & 7.068 & 5.63 & 4.432 & 3.21 & 2.149 & 1.37 & 2.627 \\
\hline 5 & 168.7812195 & -61.2629204 & 5.10 & 2.825 & 1.30 & 1.323 & 1.17 & 2.016 & 11.80 & 9.528 & 8.00 & 6.703 & 5.38 & 4.209 & 3.36 & 4.067 \\
\hline 6 & 168.7785034 & -61.2624550 & 5.01 & 2.776 & 1.26 & 1.284 & 1.12 & 1.968 & 9.80 & 7.562 & 6.05 & 4.786 & 3.50 & 2.385 & 1.53 & 2.727 \\
\hline 7 & 168.7825775 & -61.2605515 & 5.17 & 2.879 & 1.33 & 1.347 & 1.11 & 1.952 & 12.37 & 10.080 & 8.53 & 7.201 & 5.85 & 4.742 & 3.90 & 4.346 \\
\hline 8 & 168.7780762 & -61.2599411 & 4.83 & 2.670 & 1.22 & 1.235 & 1.09 & 1.895 & 9.31 & 7.146 & 5.69 & 4.476 & 3.24 & 2.156 & 1.35 & 2.624 \\
\hline 9 & 168.7793427 & -61.2608604 & 4.84 & 2.619 & 1.17 & 1.194 & 1.13 & 1.939 & 9.47 & 7.250 & 5.81 & 4.631 & 3.44 & 2.310 & 1.50 & 2.879 \\
\hline 10 & 168.7854614 & -61.2606812 & 5.20 & 2.936 & 1.32 & 1.342 & 1.12 & 1.973 & 10.84 & 8.578 & 6.96 & 5.642 & 4.30 & 3.176 & 2.33 & 3.204 \\
\hline
\end{tabular}

Note.- - Table 3 is presented in its entirety in the electronic edition of the Astronomical Journal. A portion is shown here for guidance regarding its form and content.

$R A$ and $D E C$ are Right Ascension and Declination of the member stars on the PC chip of WFPC2 based on the 2007 data. They are J2000 coordinates. For convenience, the color excess $E(F 220 W-F 435 W), E(F 250 W-F 435 W), E(F 330 W-F 435 W), E(F 435 W-F 555 W), E(F 555 W-F 675 W)$, and $E(F 555 W-F 814 W)$ are named $E(M U V-B), E(N U V-B), E(U-B), E(B-V), E(V-R)$, and $E(V-I)$, respectively. 
TABLE 4

MEAN COLOR EXCESSES AND EXTINCTIONS FOR MS MEMBER STARS IN THE Cluster (Without FOREGROUND REDDENING CORRECTION)

\begin{tabular}{cr}
\hline \hline Color excess \& extinction & \multicolumn{1}{c}{ Values } \\
\hline$E(F 220 W-F 435 W)$ & $4.97 \pm 0.21$ \\
$E(F 250 W-F 435 W)$ & $2.78 \pm 0.18$ \\
$E(F 330 W-F 435 W)$ & $1.31 \pm 0.12$ \\
$E(F 435 W-F 555 W)$ & $1.33 \pm 0.12$ \\
$E(F 555 W-F 675 W)$ & $1.08 \pm 0.05$ \\
$E(F 555 W-F 814 W)$ & $1.91 \pm 0.06$ \\
$A_{F 220 W}$ & $11.01 \pm 0.12$ \\
$A_{F 250 W}$ & $8.83 \pm 0.12$ \\
$A_{F 330 W}$ & $7.35 \pm 0.12$ \\
$A_{F 435 W}$ & $6.05 \pm 0.10$ \\
$A_{F 555 W}$ & $4.72 \pm 0.10$ \\
$A_{F 675 W}$ & $3.64 \pm 0.10$ \\
$A_{F 814 W}$ & $2.80 \pm 0.10$ \\
$R_{F 555 W}$ & $3.54 \pm 0.63$ \\
\hline
\end{tabular}

